\title{
ANNIHILATION OF POSITRONS IN Fe-Ni ALLOYS
}

\author{
S. SzuszkiEWICZ \\ Institute of Experimental Physics, Wrocław University \\ Maxa Borna 9, 50-204 Wrocław, Poland
}

\begin{abstract}
The effect of positron polarization on annihilation characteristics for ferromagnetic alloy samples, $\mathrm{Fe}_{64} \mathrm{Ni}_{36}, \mathrm{Fe}_{63} \mathrm{Ni}_{37}, \mathrm{Fe}_{25} \mathrm{Ni}_{75}$ and $\mathrm{Fe}_{50} \mathrm{Ni}_{50}$, has been studied. It was observed that the global two-quantum annihilation rate depends on mutual orientation of positron beam direction and the sample magnetization. The observed changes of the rate are opposite to the ones which could be expected from the effect of mutual orientation of positron and electron spins on positron backscattering. They can be explained as a result of changing of the relation between $3 \gamma$ - and $2 \gamma$-annihilation probabilities.
\end{abstract}

PACS numbers: $78.70 . \mathrm{Bj}$

Our previous results [1] have shown that the annihilation of polarized positrons can be a useful tool in studying the scattering of positrons on a sample.

It is well known that positrons, emitted from a radioactive source, have their spins partially polarized. In the $\beta^{+}$-decay process, the positron spin is predominantly parallel to the positron velocity $v_{\mathrm{p}}$ and the probability of such spin orientation is proportional to $v_{\mathrm{p}} / c$ ratio, where $c$ is the light velocity and $v_{\mathrm{p}}=\left|v_{\mathrm{p}}\right|$. In the case of annihilation of positrons with the saumple electrons, the positron polarization changes considerably as a result of both the backscattering which is due to the interaction of positrons with the sample electrons and the process of positron thermalization in the sample $[2,3]$. Nevertheless significant positron spin ordering remains up to annihilation of positrons.

When the spins of annihilating particles, i.e. positron and electron are antiparallel, a two-quantum annihilation takes place. By applying an external magnetic field to a ferromagnetic sample one can obtain the appropriate spin orientation of the sample electrons with respect to the applied magnetic field $\boldsymbol{B}$ direction (majority of electrons have their spins antiparallel to the $\boldsymbol{B}$ direction). Therefore, we can expect a difference in the angular distributions of annihilation quanta, which results from two-quantum annihilation, for two distinctly different directions of the external magnetic field: parallel and antiparallel to the direction of positron polarization.

Measurements were performed for polycrystalline $\mathrm{Fe}_{64} \mathrm{Ni}_{36}, \mathrm{Fe}_{63} \mathrm{Ni}_{37}$, $\mathrm{Fe}_{25} \mathrm{Ni}_{75}$ and $\mathrm{Fe}_{50} \mathrm{Ni}_{50}$ alloy samples. All these alloys have the same crystallographic structure of the fcc type. First two samples were from the invar region 
while the remaining ones from the permalloy region. To improve the plasticity, enabling the cold rolling of the samples, about 0.17 at.\% of $\mathrm{Mn}$ has been introduced during the preparation of the alloys. The samples were heated in vacuum at $950^{\circ} \mathrm{C}$ for 5 hours, before measurements, and then gradually cooled in order to remove the defects created during the process of the sample preparation.

The angular distributions (AD) of annihilation quanta have been measured by means of a standard long-slit annihilation spectrometer with the angular resolution of about $1.2 \mathrm{mrad}$. A sample and a source of positrons ( ${ }^{22} \mathrm{Na}$ having an activity of $25 \mathrm{mCi}$ ) were placed between electromagnet poles. The external magnetic field of $1.1 \mathrm{~T}$ was used to order electron spins in the sample and to focus positrons on the sample.

The AD measurements were performed with the magnetic field $\boldsymbol{B}$ parallel and antiparallel to the positron beam direction. In the case when $\boldsymbol{B}$ is parallel to this direction, the orientations of electron spins are predominantly antiparallel to the orientations of positron spins. Let us denote the AD measured in such case as $N_{\mathrm{a}}(\Theta)$. When $\boldsymbol{B}$ and the positron beam direction are antiparallel to each other the mutual orientations of electron and positron spins are predominantly parallel. Let us denote the AD measured for this case as $N_{\mathrm{p}}(\Theta)$.

The area under the experimental distribution is proportional to the number of processes of two-quantum annihilation of positrons with the sample electrons. For the measured distributions $N_{\mathrm{a}}(\Theta)$ and $N_{\mathrm{p}}(\Theta)$ we have obtained two different areas under the curves for each sample, as related to the same measurement time, i.e. for the same number of positrons impinging on the sample. For each investigated sample the area related to $N_{\mathrm{p}}(\Theta)$ distribution appeared to be less than that related to $N_{\mathrm{a}}(\Theta)$ one. This means that in the case when parallel spins of annihilating particles (electrons and positrons) are more probable, the rate of the two-quantum annihilation is smaller.

In order to describe the observed difference the parameter $\eta$ defined as

$$
\eta=\frac{N_{\mathrm{a}}-N_{\mathrm{p}}}{N_{\mathrm{a}}+N_{\mathrm{p}}}
$$

has been calculated. Here

$$
N_{\mathrm{a}, \mathrm{p}}=\int_{-\infty}^{\infty} N_{\mathrm{a}, \mathrm{p}}(\Theta) \mathrm{d} \Theta .
$$

In Fig. 1 we present the average value $\mu$ of the magnetic moment per alloy atom as a function of $\mathrm{Ni}$ concentration in the $\mathrm{Fe}-\mathrm{Ni}$ alloy [4-8]. In the same figure we have also shown values of the parameter $\eta$ obtained for the investigated samples. As one can see the character of the dependence of this parameter on the $\mathrm{Ni}$ concentration is similar to that of the magnetic moment.

The fact that the parameter $\eta$ is different from zero means that the change of the mutual orientation of magnetic field and positron beam directions causes the change of the probability of two-quantum annihilation for positrons impinging on the sample. For positrons reaching the sample there are three possibilities of their further fate: backscattering from the sample, two-quantum annihilation and three-quantum annihilation. According to Bhabha results [9] the backscattering of positrons from the sample should be greater when the antiparallel mutual orientation of positron and electron spins is predominant. It means that the expected 


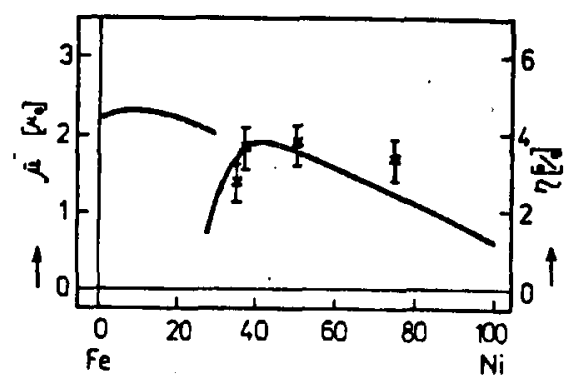

Fig. 1. The dependence of $\eta$ and $\mu$ on $\mathrm{Ni}$ concentration in $\mathrm{Fe}-\mathrm{Ni}$ alloys.

contribution to $\eta$ value due to the change of positron backscattering when going from the one magnetic field direction to the other should have the opposite sign to that observed for $\eta$ value. Thus the obtained results can be explained by assuming that the probability of three-quantum annihilation is considerably greater when the parallel mutual orientation of positron and electron spins is more probable than the antiparallel one. The role of the effect of the direction of sample magnetization on positron backscattering is, if any, very small for the studied materials.

The work was supported by the Wroclaw University (gw 12/94).

\section{References}

[1] M. Szuszkiewicz, S. Szuszkiewicz, J. Chojcan, Phys. Status Solidi B 141, K79 (1987).

[2] L.P.L.M. Rabou, Rep. ECN-129, Netherland Energy Research Found, Research Found, Petten 1983.

[3] M. Sob, S. Szuszkiewicz, M. Szuszkiewicz, Phys. Stalus Solidi B 123, 649 (1984).

[4] C.G. Shull, M.K. Wilkinson, Phys. Rev. 97, 304 (1955).

[5] M.F. Collins, J.B. Forsyth, Philos. Mag. 8, 401 (1963).

[6] M.F. Collins, G.G. Low, Proc. Phys. Soc. 86, 535 (1965).

[7] M.F. Collins, R.V. Jones, R.D. Lowole, J. Phys. Soc. Jpn. 17, B-III, 19 (1962).

[8] J. Crangle, G.C. Hallam, Proc. R. Soc. Lond. A Math. Phys. Sci. 272, 119 (1963).

[9] H. Frauenfelder, R. Steffen, in: $\alpha, \beta$ and $\gamma$ Ray Spectroscopy, Vol. 2, Ed. K. Siegbahn, North-Holland, Oxford 1979, p. 1444. 